\title{
Self-testing of sigma-delta MEMS sensors using BIMBO
}

\author{
J. Juillard \\ SUPELEC, SSE \\ Gif-sur-Yvette, France \\ jerome.juillard@supelec.fr
}

\author{
E. Colinet \\ CEA-LETI, MINATEC \\ Grenoble, France \\ eric.colinet@cea.fr
}

\begin{abstract}
This paper deals with the test of sigma-delta MEMS sensors thanks to BIMBO, an identification method based on binary observations. We show how this method may be used in order to estimate the parameters of the sensing cell and of the associated analog electronics. The principle of the method and its theoretical properties are briefly presented and its pros and cons are discussed. Two approaches for the test of sigma-delta sensors are then presented and compared.
\end{abstract}

\section{INTRODUCTION}

Sigma-delta MEMS (mostly inertial sensors) have been a large field of interest over the past few years [1][2]. Such sensors are feedback-controlled systems where the feedback is a high-frequency binary signal which represents, in an averaged sense, the system's input (e.g. the acceleration). There are many advantages to this approach such as linearization of the (electrostatic) feedback forces, enhancement of the sensor's dynamic range, digital measurement and easy VLSI implementation [3]. A typical sigma-delta sensor architecture is shown in Fig. 1.

It can be shown [4-5] that the resolution obtained with advanced architectures such as those of Fig. 1 is directly linked to the choice of the corrector $G(z)$ which appears in the feedback loop. However, even choices based on robust control considerations [4] do not guarantee the optimal performance of the system, nor can they always cope with the large dispersions, mostly due to variations in the fabrication process or environmental fluctuations, which affect MEMS devices. As a consequence, a self-testing routine is a desirable feature of such architectures: if the parameters of the sensing cell and of the associated analog electronics are correctly estimated, the coefficients of $G(z)$ can be automatically adjusted, in order to ensure a better performance. The same goes for the decimation/filtering stage.

One of the major difficulties in implementing a selftesting routine in a microelectronic device is to keep the cost of the design and of the integration of the method as small as possible. Therefore, classical system identification methods
[6], which rely on high-resolution ADCs are usually ruled out because they are uneconomical in terms of design and integration costs,. Although it may be possible, in some cases, to develop low-cost ad hoc self-test methods, a more systematic approach should be preferred, if only to reduce the design time of a given device. The BIMBO (Basic Identification Method using Binary Observations) method has recently been developed in order to address these issues [7-8].

The present paper deals in detail with the use of BIMBO in the context of sigma-delta MEMS sensors. We show how the BIMBO method can be used for estimating the parameters of sigma-delta sensor architectures. In the first part of the paper, we expose the principle of the BIMBO method, its pros and cons and in what circumstances it should be used. We also give a brief review of the theoretical properties of this method. We then go on to show how BIMBO can be applied to the identification of a sigma-delta MEMS sensor and illustrate this approach in the case of an accelerometer.

\section{BASIC IDENTIFICATION METHOD USING BINARY OBSERVATIONS}

BIMBO is an identification method that has been developed for responding to the issues of self-testing in a context of integrated electronics and MEMS, as mentioned in the introduction. The only analog component it requires is a comparator, which is used as a 1-bit ADC. As a consequence, the integration of this method has a very low cost, which makes it attractive for the self-test of integrated MEMS sensors and analog ICs. Another advantage of the approach is that parasitic capacitances can be kept very small. Yet another attractive feature is that BIMBO can be used with binary test signals and, thus, a minima, requires only a 1-bit DAC. This last characteristic makes BIMBO a natural candidate for the self-test of sigma-delta MEMS sensors because these architectures usually include a 1-bit ADC, as well as a 1-bit DAC. Sigma-delta architectures are well-adapted to capacitive MEMS sensors because binary 


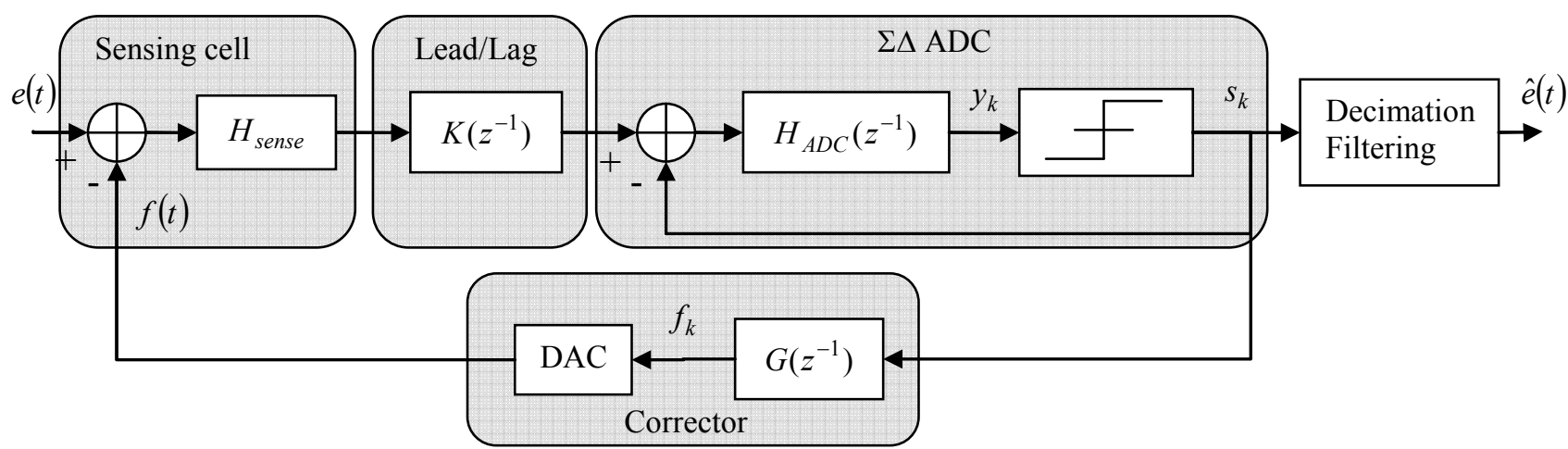

Figure 1. Typical sigma-delta sensor architecture. In capacitive MEMS applications, sigma-delta binary feedback is often used. The sensing cell, lead/lag filter and ADC filter are subject to dispersions in the fabrication process and, to a lesser extent, to variations in the environmental conditions.

control signals are not distorted by electrostatic actuation forces.

The major drawback of BIMBO is that, since no amplitude measurement is made, there always remains one indeterminate static gain unknown in the tested system. Also, this approach usually requires more data points than classical identification methods, based on amplitude measurements however, this is compensated by the fact that only binary data must be stored.

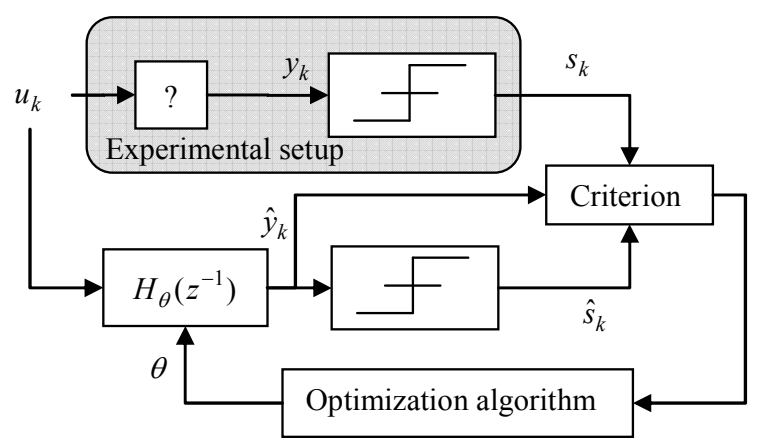

Figure 2. Block diagram of the BIMBO method. Using only binary observations, a parametric model of the real system is built so as to maximize the similarity between $s_{n}$ and $\hat{s}_{n}$.

\section{A. Principle}

The principle of BIMBO is the following (see Fig. 2 for notations):

- generate signal $u_{k}$, such as a white noise. This signal can be a one-bit signal so that the DAC can be implemented with ease.

- measure the system's output $y_{k}$ using a comparator, i.e. measure $s_{k}=S\left(y_{k}\right)$

- using a discrete parametric model of the system, compute the estimated time response $\hat{y}_{k}$ and $\hat{s}_{k}=S\left(\hat{y}_{k}\right)$
- adjust the parametric model of the discrete system so that a correct estimation $\hat{y}_{k}$ is produced.

An optimization algorithm is necessary for this last step. Letting $\boldsymbol{\theta}$ be the unknown system parameters, the (continuous) criterion that must be minimized is of the form:

$$
J(\hat{\boldsymbol{\theta}})=\frac{\sum_{1}^{N}\left(s_{k}-S\left(\hat{y}_{k}\right)\right)^{2} \hat{y}_{k}{ }^{2}}{\sum_{1}^{N} \hat{y}_{k}{ }^{2}},
$$

where $N$ is the number of samples and $S($.$) is the sign$ function. In case there is no model error, there exists a zone in $\boldsymbol{\theta}$-space for which the criterion is zero. This zone becomes smaller as the number of data points increases. Note that no approximation of the ADC is being made, as opposed to classical identification methods [6,9-11], which are based either on an additive white noise model of the $\mathrm{ADC}$ or on a describing function approximation. Finally, note that this method can be used in a completely deterministic framework, without any assumptions concerning $u_{k}$ (intuitively, the method should work as long as $u_{k}$ is spectrally rich enough).

\section{B. Theoretical properties of BIMBO}

In order to establish the theoretical properties of BIMBO, one has to place oneself in a probabilistic framework. First of all, under the assumption that $u_{k}$ is a gaussian white noise and that the unknown system is linear, one can prove that the limit of (1) as the number of samples goes to infinity is:

$$
J(\hat{\boldsymbol{\theta}})=1-\frac{2}{\pi}\left(r \sqrt{1-r^{2}}+\sin ^{-1}(r)\right),(2)
$$




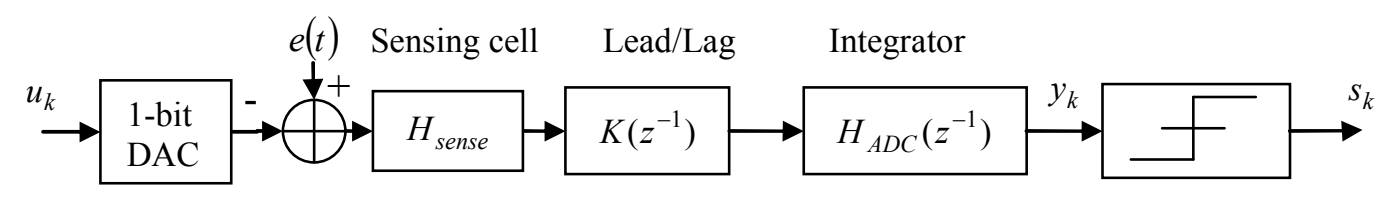

Figure 3. Open-loop setup. The external perturbation is supposed to be constant.

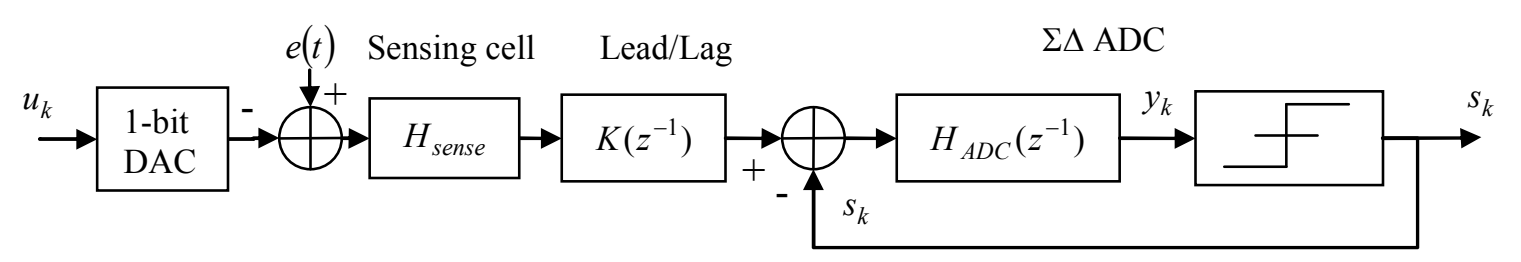

Figure 4. Closed-loop setup.

where $r$ is the correlation coefficient between the unknown system with parameters $\boldsymbol{\theta}$ and the estimated system with parameters $\hat{\boldsymbol{\theta}}$. Under simple assumptions on $r$, it is then simple to prove that, for an infinite number of samples, the zone where the criterion is zero reduces to a point and that, in the neighborhood of this point, the proposed criterion becomes convex. As a consequence, a simple gradient descent is all that is required to minimize (1), provided a good starting point is known. This starting point may be found without prior information by using the techniques described in [12]. For example, supposing $u_{k}$ is a gaussian white noise, an estimate of the unknown system impulse response $\hat{h}_{p}$ is given by:

$$
\hat{h}_{p} \propto \sin \left(\frac{\pi}{2} \hat{C}_{s u}(p)\right)
$$

where $\hat{C}_{s u}(p)$ is an empirical estimate of the crosscovariance function of the system's input and output. If $u_{k}$ is a binary white noise, the impulse response is given by:

$$
\hat{h}_{p} \propto \frac{\operatorname{erf}^{-1}\left(\hat{C}_{s u}(p)\right)}{\sqrt{1+2\left(\operatorname{erf}^{-1}\left(\hat{C}_{s u}(p)\right)\right)^{2}}}
$$

A lower-order model, such as an ARX model, can then be fitted to this estimation of the impulse response.

\section{IDENTIFICATION OF A SIGMA-DELTA MEMS SENSOR USING BIMBO}

BIMBO can be used in a variety of ways to estimate the parameters of a sigma delta MEMS sensor: the preferable method is to use the open-loop approach described in subsection III-A. This method should be favored because, provided the unknown system is linear, the properties presented in subsection II-B hold and can be put to use. However, this approach is completely offline and may not be easy to implement in some contexts. We show in section III$\mathrm{B}$ how to use BIMBO when the feedback loop of the sigmadelta sensor cannot be opened.

\section{A. Open-loop approach}

Intuitively, the open-loop approach is the simplest way to guarantee the whiteness of the excitation signal and, thus, to ensure a correct identification of the unknown system. This approach consists in opening the feedback loop(s) of the sensing cell and in applying a known excitation (control) signal to the system input, while supposing zero perturbation. The 1-bit ADC output is then measured and BIMBO can be applied. The 'zero perturbation' hypothesis is necessary in many sigma-delta sensors because of the presence of the integrators in the feedforward path. However, this restriction may be slackened thanks to the leaks that occur in the integrators: one may then suppose that the unknown perturbation is a constant and use BIMBO to estimate its value as well the system parameters. In order to illustrate this approach, consider the accelerometer sensing cell and electronics of Fig. 3. The simulation is performed in three steps: some nominal parameters are assigned to the different blocks (sensing cell, phase lead, integrator leaks and constant value of the perturbation). The system output $s_{n}$ is then calculated for a known binary input signal $u_{n}$. Criterion (1) is then minimized in four sub-steps:

- find a starting guess for the unknowns.

- minimize (1) with a "small" set of data in order to find the open-loop system impulse response and the value of the perturbation.

- fit a lower-order (ARX) model to the estimated impulse response.

- use the lower-order model as a new starting guess for minimizing (1) with a "large" set of data. 
A simple way to find a starting guess for the value of the nonzero perturbation is to suppose that $y_{n}$ is gaussian, as in [12], and to determine an estimate of the impulse response with (4). An estimate of the value of the perturbation can then be inferred from the probability that $s_{n}$ be positive. Typical results are shown in Fig. 5.
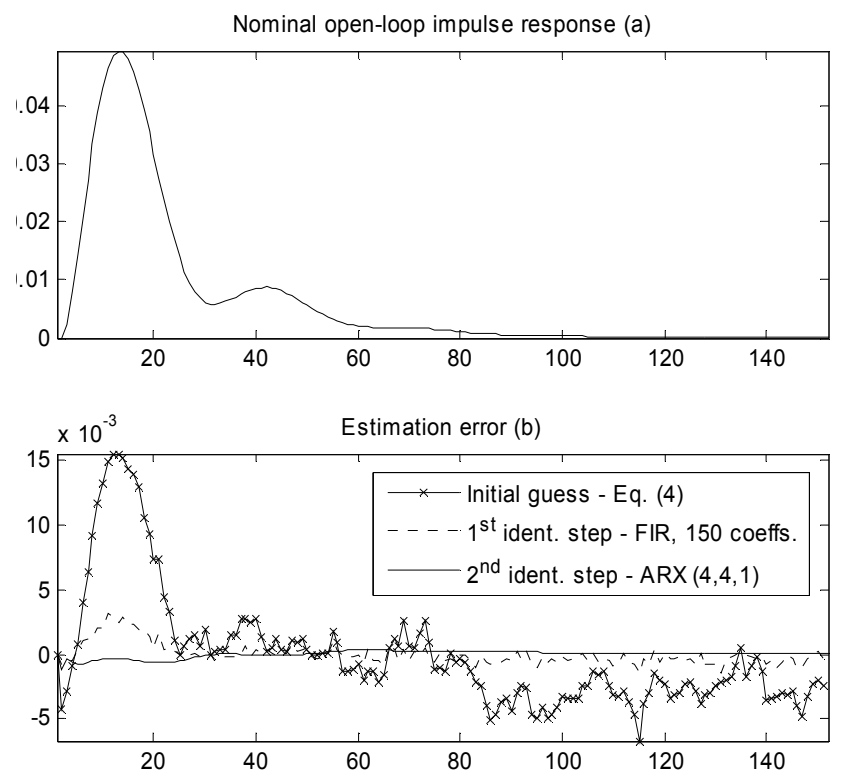

Figure 5. Nominal impulse response of the open-loop system (a) and absolute estimation error (b) at different steps of the identification procedure. In the open-loop approach, $2^{14}$ samples of $s_{n}$ and $u_{n}$ are used to identify 150 coefficients of the impulse response. After 10 gradient steps, a $(4,4,1)$ ARX model is fitted to the estimated FIR filter and $2^{16}$ samples of $s_{n}$ and $u_{n}$ are used to refine this result. Similar results can be obtained with the closed loop approach.

\section{B. Closed-loop approach}

In case the feedback loops cannot be opened, it is possible to identify the whole system without having to provide an exterior excitation signal. However $s_{n}$, which plays the role of excitation, is not white and this usually leads to poorer results than with the open-loop approach. It is then preferable to partially open the outermost loop (as in Fig. 4) and to identify the system with the following heuristic:

- suppose the integrator stage is nominal and either use the sigma-delta filtered and decimated output to estimate the sensing cell, phase lead and perturbation parameters or minimize (1).

- use the resulting parameters to determine the input of the integrator stage.

- minimize (1) with respect to the integrator stage parameters.
- $\quad$ iterate the process to refine the estimation.

However accurate, this approach is less systematic than the open-loop approach and requires more computational effort.

\section{CONCLUSION}

The main feature of the BIMBO method is that it only requires binary observations (and binary inputs): it is thus well-suited to the constraints of integrated microelectronics and it is a particularly good choice for the test of sigma-delta (MEMS) sensors. After reviewing the fundamental theoretical properties of the method, we described two different ways to use it the context of sigma-delta sensors. Both have already been used and validated experimentally. Future work includes online identification, nonlinear system identification and studying the theoretical properties of the method for closed loop systems.

\section{REFERENCES}

[1] M. Lemkin, B. Boser, "A three-axis micromachined accelerometer with a CMOS position-sense intefrace and digital offset-trim electronics", IEEE Journal of Solid State Circuits, vol. 34, pp. 456468, 1999

[2] M. Kraft, Closed loop digital accelerometer employing oversampling conversion, Ph. D. Thesis, University of Coventry, U.K., 1997

[3] M. Kraft, C.P. Davies, T.G. Hesketh, "Closed loop silicon accelerometers", IEE Proceedings - Circuits, Devices and Systems, vol. 145 , no. 5 , pp. $325-331,1998$

[4] E. Colinet, J. Juillard, S. Guessab, R. Kielbasa, "Resolution enhancement of a sigma-delta micro-accelerometer using signal prediction", International Conference on MEMS, NANO and Smart Systems, Banff, 2004, pp. 409-413

[5] C. Condemine, Contributions à la conception de microsystèmes sigma-delta asservis, Ph. D. Thesis, INSA Lyon, 2001

[6] L. Ljung, System identification - theory for the user, Prentice Hall, 1999

[7] E. Colinet, Nouvelles architectures et méthodes de conception de microsystèmes sigma-delta et de microsystèmes résonants, $\mathrm{Ph} . \mathrm{D}$. Thesis, Université Paris-Sud Orsay, France, 2005

[8] E. Colinet, J. Juillard, "An identification method based on binary observations", submitted to IEEE Transactions on Automatic Control (2006)

[9] Astrom K. J., Hagglund T. H. , "Automatic tuning of simple regulators with specifications on phase and amplitude margins", Automatica, vol. 20, n ${ }^{\circ}$, pp. 645-651, 1984

[10] D. Vazquez et al., "On chip evaluation of oscillation based test output signals for switched capacitor circuits", Journal of Analog Integrated Circuits and Signal Processing, vol. 33, n², pp. 201-211, 2002

[11] Q.-G. Wang, T.H. Lee, C. Lin, Relay feedback: analysis, identification and control, Springer, 2003

[12] J. Juillard, E. Colinet, "Initialization of the BIMBO self-test method using binary inputs and outputs", submitted to Conference on Decision and Control 2007 